\title{
Activités
}

17-1 | 2020

IA, robotique, automatisation : quelles évolutions pour l'activité humaine?

\section{“Collectifs de travail en ingénierie aéronautique : comment soutenir et développer l'activité collective en contexte de transformation organisationnelle et digitale ?" [Résumé]}

Thèse de Doctorat en Psychologie. Mention : Psychologie du travail et des organisations. Université Lyon 2. 16 décembre 2019

\section{Pauline Crouzat}

\section{OpenEdition}

Journals

Édition électronique

URL : http://journals.openedition.org/activites/5167

DOI : 10.4000/activites.5167

ISSN : $1765-2723$

Éditeur

ARPACT - Association Recherches et Pratiques sur les ACTivités

\section{Référence électronique}

Pauline Crouzat, «"Collectifs de travail en ingénierie aéronautique : comment soutenir et développer l'activité collective en contexte de transformation organisationnelle et digitale ?" [Résumé] », Activités [En ligne], 17-1 | 2020, mis en ligne le 15 avril 2020, consulté le 23 septembre 2020. URL : http:// journals.openedition.org/activites/5167 ; DOI : https://doi.org/10.4000/activites.5167

Ce document a été généré automatiquement le 23 septembre 2020.

\section{(1) $\odot \Theta \Theta$}

Activités est mis à disposition selon les termes de la licence Creative Commons Attribution - Pas d'Utilisation Commerciale - Pas de Modification 4.0 International. 


\section{"Collectifs de travail en ingénierie aéronautique : comment soutenir et développer l'activité collective en contexte de transformation organisationnelle et digitale?" [Résumé]}

Thèse de Doctorat en Psychologie. Mention : Psychologie du travail et des organisations. Université Lyon 2. 16 décembre 2019

Pauline Crouzat

\section{RÉFÉRENCE}

Pauline Crouzat (2019). Collectifs de travail en ingénierie aéronautique : comment soutenir et développer l'activité collective en contexte de transformation organisationnelle et digitale?. Thèse de Doctorat en Psychologie. Mention : Psychologie du travail et des organisations. Université Lyon 2. Soutenue publiquement le 16 décembre 2019

\section{NOTE DE L'ÉDITEUR}

\section{Jury de thèse}

- Marc-Éric Bobillier Chaumon, Professeur en Psychologie du travail. Conservatoire National des Arts et Métiers (Directeur)

- Sandrine Caroly, Professeur des Universités en Ergonomie. Université Grenoble Alpes (Rapporteur)

- Anne-Marie Costalat-Founeau, Professeure des Universités Emérite en Psychologie 
sociale et du travail. Université de Montpellier 2 (Examinatrice)

- Claire Hribar, Ingénieur (Membre invité)

- Katia Kostulski, Professeure des Universités en Psychologie du Travail. Conservatoire

National des Arts et Métiers, Paris (Rapporteur)

- Philippe Sarnin, Professeur des Universités en Psychologie du Travail et des

Organisations. Université Lyon 2 (Examinateur, président du Jury)

\section{Introduction}

1 Cette recherche a été réalisée au sein de l'ingénierie d'une entreprise aéronautique. Elle répond à une commande de l'entreprise qui souhaite développer la collaboration au sein des services d'ingénierie. Il s'agit de déterminer quelles peuvent être les ressources et les contraintes socio-organisationnelles d'une activité collective efficiente et signifiante pour les salariés. Et, comment, d'un point de vue psychologique et sociocognitif, favoriser le développement de cette dernière. Notre compréhension du terrain s'appuie sur une triangulation théorique s'appuyant sur la clinique de l'activité (Clot, 2002), la clinique de l'usage (Bobillier Chaumon, \& Clot, 2016) et l'approche ergonomique (Caroly, 2010). La démarche globale de cette recherche-intervention se base sur une méthodologie mixte (qualitative et quantitative) impliquant les acteurs de l'ingénierie comme co-chercheurs.

\section{Contexte et cadrage}

2 Après une présentation de l'entreprise étudiée et de l'organisation globale et matricielle de ses services d'ingénierie, cette thèse aborde dans un premier temps les modèles d'analyse de l'activité existants (Engerström, 1987; Leplat, 1997, 2006; Rabardel, \& Vérillon, 1985; Vygotski, 1925/2003). Elle présente ensuite la notion d'activité collective (Caroly \& Barcellini, 2013 ; Clot, 2010), objet de la recherche, avant de définir ses composantes : le travail collectif et le collectif de travail (Caroly, 2010), mais aussi d'aborder la notion de collectif transverse (Poret, 2015). Un état des lieux concernant la littérature et les travaux scientifiques précisant l'intérêt de développer l'activité collective dans une organisation y sont également abordés: en termes de santé (Machado, 2015 ; Sarnin, Caroly, \& Douillet, 2011), en termes de compétences et d'intelligence collective (Krohmer, 2011), d'innovation ou encore de performance. Nous présentons ensuite les ressources psychosociales et socio-cognitives connues de l'activité collective à savoir les différentes formes de synchronisation qui soutiennent le travail collectif, mais aussi les opportunités de dialogue et de controverse qui soutiennent la structuration et la consolidation des collectifs de travail (Kostulski, 2015 ; Kostulski, \& Clot, 2007) ainsi que les genres professionnels impliqués (Clot, 2000). Nous précisons également la nature de l'activité au sein de l'ingénierie et détaillons le système d'instruments qui la conditionne. Nous évoquons la nature des tâches, mais aussi le type d'activité pouvant avoir cours en distinguant notamment les activités fonctionnelles des activités métafonctionnelles (Falzon, 1994) avant de définir les notions d'outil et d'instruments (Rabardel, 1995) puis de caractériser la plupart des outils socio-techniques qui portent l'activité au sein de l'ingénierie (Gilbert, \& Chiapello, 2012). En effet, selon leurs propriétés, les outils (digitaux, cognitifs) à disposition peuvent produire des effets sur l'activité collective, notamment en affectant 
les modalités d'interaction entre les acteurs (et donc les collectifs de travail) : ce sont bien ces dernières qu'il s'agit d'interroger au cours de notre recherche qui s'articule autour de trois projets itératifs menés simultanément au sein de l'entreprise.

\section{Déterminer la nature de l'instrumentation de l'activité}

3 Au cours d'un premier projet d'étude (projet A), nous définissons la structure socioorganisationnelle du système d'ingénierie étudié. L'objectif est d'évaluer la nature de l'instrumentation (outils socio-techniques et initiatives) déployée pour supporter l'activité collective. L'ensemble des résultats de ce projet A, basé sur des observations, des entretiens, mais aussi sur l'analyse de documents internes relatifs à l'organisation du travail, démontre que les initiatives et les outils socio-techniques de gestion et de communication dévolus au développement du travail collectif sont nombreux et, pour la plupart, utilisés par les salariés. L'hypothèse apparue au début de ce projet se confirme pour le travail collectif de la population: ce-dernier est fortement instrumenté voire hyper-instrumenté. La satisfaction et l'intérêt des salariés concernant les temps de rencontre physique et de dialogue confirment néanmoins leur besoin d'espaces dialogiques et de temps dédiés à l'élaboration et à la réflexion collectives. En un même temps, avec la multiplication et le croisement d'instruments socio-techniques (chronométrage, supports de travail pré-remplis, outils digitaux, processus à appliquer), les propositions et les idées se croisent et se succèdent alors que le temps nécessaire dédié à leur exploitation, à leur centralisation ou à leur compréhension apparaît comme considérablement réduit. Le dialogue et l'activité conversationnelle semblent en diminution constante or, ils constituent une ressource indispensable à la structuration et/ou à la consolidation des collectifs à l'œuvre (Kostulski, 2005).

\section{Déterminer les ressources psychologiques et sociocognitives de l'activité}

4 Le second projet d'étude (projet B) a pour objectif de déterminer les contraintes et les ressources psychologiques et sociocognitives (Caroly, 2010) d'une activité collective efficiente et signifiante pour les salariés. Ce second projet d'étude retrace l'analyse du vécu et des représentations ayant cours chez les acteurs concernés afin de mieux comprendre leurs besoins ainsi que ce qui soutient et/ou contraint les composantes de leur activité collective. Les résultats des études, basées sur la technique des incidents critiques (Flanagan, 1954) et sur des entretiens semi-directifs, ont approfondi les résultats obtenus au cours du projet $\mathrm{A}: \mathrm{au}$ sein du système étudié, on ne retrouve effectivement pas toutes les conditions d'émergence des collectifs de travail inhérentes à une organisation de travail se basant sur les métiers. Dans l'organisation du travail étudiée ici, la division matricielle, ajoutée à l'hyper-instrumentation de l'activité, réduit de façon conséquente les opportunités d'ajustement et de travail humain. Conséquence : ici, le genre professionnel n'est plus lié à un métier ou à une fonction spécifique, mais il est à construire et reconstruire indéfiniment. Il en va de même pour les styles d'action individuels qui peuvent en découler (appropriation difficile de 
l'activité): les modalités d'introjection des dynamiques collectives dans les tâches individuelles sont complexifiées. L'hypothèse sous-tendant ce projet d'étude s'est ici confirmée: la réorganisation fréquente de l'activité sur un mode matriciel (par fonctions et par projets) couplée à l'hyper-instrumentation de l'activité fragilise les genres professionnels et les référentiels communs nécessaires à la structuration des collectifs de travail. Ces derniers ne semblent d'ailleurs pas consistants. Ils ne correspondent pas aux équipes et aux groupes de travail prescrits, y compris transversaux (ils ne "s'incarnent pas »). On assiste par ailleurs à une nouvelle activité méta-fonctionnelle croissante (dans une activité initiale déjà souvent de type métafonctionnel) avec un investissement de temps et d'énergie (individuels et collectifs) consacrés directement à la structuration de l'écosystème de travail. Cette part du travail est identique chez tous les acteurs rencontrés, quels que soient leur métier initial, leur fonction, leur mission ou leurs tâches, que leur activité principale soit fonctionnelle ou méta-fonctionnelle (Falzon, 1994). Ce travail est essentiellement constitué par la recherche et le don d'informations: la circulation de données. La charge est proportionnelle au nombre d'interfaces organisationnelles à intégrer dans l'ensemble de l'activité individuelle: or, dans une organisation matricielle de l'activité, ces interfaces ne cessent de se multiplier.

\section{In-ter-venir : réinstaurer de la réflexivité dans le travail}

5 En dernier lieu, nous présentons notre projet $\mathrm{C}$ qui concerne les dispositifs que nous avons co-élaboré et déployé avec et auprès des salariés dans le but d'instrumenter l'activité collective. Ce projet se situe du côté de l'intervention psychologique du point de vue des ressources à déployer pour soutenir l'activité collective. Notre intervention a consisté en la mise en place d'un dispositif intermédiaire susceptible de soutenir l'activité collective auprès de 3 équipes rencontrant des " difficultés de collaboration ». Ce dispositif, dont nous détaillons le déploiement au cours de ce projet $\mathrm{C}$, se compose d'outils sémiotiques (Vergnaud, 2000), couramment utilisés par les salariés (mais détournés de leur usage habituel) et d'un atelier de travail laissant une place majeure à une activité de type dialogique et médiatisé par nos soins. Il a été élaboré grâce aux observations effectuées au cours des projets A et B. La conception et la mise en œuvre de cet instrument s'appuient sur la clinique de l'usage (Bobillier Chaumon, \& Clot, 2016) et sur les ressources connues du développement de l'activité collective en clinique de l'activité, notamment sur l'activité dialogique (Clot, 2008 ; Clot \& Faïta, 2000 ; Crouzat, \& Bobillier Chaumon, 2018; Kostulski \& Clot, 2007). L'instauration d'un dispositif dialogique devait ici favoriser au sein des équipes la réflexivité, la prise de conscience de sa place et de son rôle en complémentarité des pairs. La prise en compte des dimensions d'analyse des usages des technologies (Bobillier Chaumon, 2013, 2017) que nous avons appliquées ici à la conception de notre dispositif a favorisé l'acceptation de notre démarche par l'ensemble des acteurs qui ont participé à toutes les phases du dispositif. Concernant l'apport et les bénéfices psychologiques du dispositif dialogique déployé, nous retiendrons essentiellement l'amorce d'une dynamique jusqu'ici manquante : celle d'une réflexivité et d'un dialogue communs. Les exercices proposés ont en effet privilégié les ressources (dialogue, repérage mutuel) afin de construire et de stabiliser les genres professionnels (Clot, 2000) qui ne se structurent plus autour des 
métiers. Notre in-ter-vention a autorisé une rupture momentanée d'une activité continue de simple formalisation et transmission d'informations. Et cette rupture a permis une prise de conscience collective, tacite ou non, au sein des trois équipes : la seule circulation de l'information ne permet pas de partager le réel de l'activité de chacun. D'un point de vue clinique, cette intervention a réinstauré la dimension singulière du travail, en permettant l'expression (et non l'information) du collègue, le récit de son activité, la réflexion, l'étonnement et la confrontation au réel de l'activité (Clot, 2000) de ses collègues soit des éléments indispensables à la constitution d'une histoire commune et d'une collaboration efficiente, efficace et qui fait sens pour les acteurs impliqués.

\section{Conclusion}

6 L'ensemble des résultats de ces recherches nous a permis d'identifier trois phénomènes impactant directement la qualité de l'activité collective : l'hyper-instrumentation du travail par des outils digitaux et processuels qui nécessitent un temps d'usage de plus en plus long ; la modification des genres professionnels avec la dissolution des métiers au profit de rôles et fonctions qui modifient fortement les conditions de repérage des salariés, mais aussi la perception de leur activité ; enfin, une modification croissante du contenu du travail, individuel et collectif, qui évolue de plus en plus vers une activité méta-fonctionnelle de structuration permanente du travail (parfois, au détriment même de l'objet initial de l'activité). Enfin, notre discussion et notre conclusion mettent en lumière les enjeux scientifiques et pratiques, mais aussi les perspectives de recherche et d'intervention qui découlent de ces phénomènes observés.

\section{BIBLIOGRAPHIE}

Bobillier-Chaumon, M-E. (2013). Conditions d'usage et facteurs d'acceptation des technologies dans l'activité : questions et perspectives pour la psychologie du travail. Mémoire pour l'habilitation à diriger des recherches. Université de Grenoble 2.

Bobillier-Chaumon, M.-E. (2017). Transformation numérique et charge de travail : comprendre les liens. In F. Martini (Ed.), Les Cahiers des RPS (pp. 28-30). Paris : Ministère du travail.

Bobillier-Chaumon, M.-E., \& Clot, Y. (2016). Clinique de l'usage. Les artefacts technologiques comme développement de l'activité. Activités, 13(2). https://doi.org/10.4000/activites.2897

Caroly, S. (2010). Activité collective et la réélaboration des règles : des enjeux pour la santé au travail. Bordeaux : Université Victor Segalen.

Caroly, S., \& Barcellini, F. (2013). Le développement de l'activité collective. P. Falzar (Ed.), Ergonomie constructive (pp. 33-46). Paris : PUF.

Clot, Y. (2000). La fonction psychologique du collectif. In A. Weill-Fassina, \& T. H. Benchekroun (Eds.), Le travail collectif. Perspectives actuelles en ergonomie (pp. 272-286). Toulouse : Octarès. 
Clot, Y. (2002). Clinique de l'activité et répétition. Cliniques méditerranéennes, 66, 31-53.

Clot, Y. (2008). Travail et pouvoir d'agir. Paris : Presses Universitaires de France.

Clot, Y. (2010). Le travail à cœur. Pour en finir avec les risques psychosociaux. Paris : La Découverte.

Clot, Y., \& Faïta, D. (2000). Genres et styles en analyse du travail. Concepts et méthodes. Travailler, $4,7-42$.

Crouzat, P., \& Bobillier Chaumon, M.-E. (2018). Recherche-intervention : acceptation située et instauration d'une activité dialogique dans l'élaboration d'un collectif de travail. In A. V. Daele, C. Hellemans, \& A. Casini (Eds.), Bien-être et diversité des situations de travail. Tome 2 : Santé psychologique et dynamiques de travail (pp. 9-21). Paris : L'Harmattan.

Engeström, Y. (1987). Learning by expanding: An activity-theoretical approach to developmental research. Helsinki, Finland: Orienta-Kosultit Oy.

Falzon, P. (1994). Les activités méta-fonctionnelles et leur assistance. Le Travail Humain, 57(1), 1-23.

Flanagan, J. C. (1954). The critical incident technique. Psychological Bulletin, 51(4), 327-358.

Gilbert, P., \& Chiapello, E. (2012). Les outils de gestion : producteurs ou régulateurs de la violence psychique au travail ? Le Travail Humain, 75(1), 1-18.

Kostulski, K. (2005). Activité conversationnelle et activité d'analyse : l'interlocution en situation de coanalyse du travail. In L. Filliettaz, \& J.-P. Bronckart Eds.), L'analyse des actions et des discours en situation de travail (pp. 57-75). Louvain-la-Neuve : Peeters.

Kostulski, K., \& Clot, Y. (2007). Interaction et migration fonctionnelle : un développement en autoconfrontation croisée. Psychologie de l'interaction, 23-24, 73-109.

Krohmer, C. (2011). Le couple DG-DRH à l'épreuve des démarches compétences. Cachan : Lavoisier.

Leplat, J. (1997). Regards sur l'activité en situation de travail. Contribution à la psychologie ergonomique. Paris, PUF.

Leplat, J. (2006). La notion de régulation dans l'analyse de l'activité. Pistes, 8(1).

Machado, T. (2015). La prévention des risques psychosociaux. Rennes : PUR.

Poret, C. (2015). Concevoir pour le pouvoir d'agir ensemble d'un collectif transverse : le cas de la relation de service dans le domaine commercial. Paris : Université Paris VIII.

Rabardel, P. (1995). Les hommes et les technologies, une approche cognitive des instruments contemporains. Paris : Armand Colin.

Rabardel, P., \& Verillon, P. (1985). Relations aux objets et développement cognitif. In Actes des septièmes journées internationales sur l'éducation scientifique, Chamonix.

Sarnin, P., Caroly, S., \& Douillet, P. (2011). Contre les « risques » psychosociaux, quel débat sur l'activité ? Le Travail Humain, 74(4), 309-320).

Vergnaud, G. (2000). Lev Vygotski. Pédagogue et penseur de notre temps. Paris : Hachette.

Vygotski, L. (1925/2003). La conscience comme problème de la psychologie du comportement. In L. Vygotski (Ed.), Conscience, inconscient, émotions (pp. 61-94). Paris : La Dispute. 


\section{AUTEURS}

\section{PAULINE CROUZAT}

École Doctorale : Sciences de l'éducation, psychologie, information et communication, Institut de Psychologie, Laboratoire : GRePS 